\title{
A promoção de saúde bucal no contexto do ambiente escolar primário: revisão bibliográfica
}

\author{
A promotion of oral health in the context of the primary school environment: bibliographic review \\ La promoción de la salud bucal en el contexto del entorno de la escuela primaria: revisión de la
}

\section{literatura}

Recebido: 03/09/2021 | Revisado: 10/09/2021 | Aceito: 12/09/2021 | Publicado: 13/09/2021

Antonio Gonçalves Oliveira Neto ORCID: https://orcid.org/0000-0003-0017-3756

Faculdade Pitágoras de Imperatriz, Brasil. E-mail: netoo98@hotmail.com

Elisama de Oliveira Mendes ORCID: https://orcid.org/0000-0003-3248-3927

Faculdade Pitágoras de Imperatriz, Brasil

E-mail: elisamaoliveira81@outlook.com

Caroline Rodrigues Thomes

ORCID: https://orcid.org/0000-0003-3522-5368

Universidade Federal do Espírito Santo, Brasil

E-mail: carolthomesodonto@gmail.com

Samuel da Silva Lima

ORCID: https://orcid.org/0000-0003-1493-9206

Faculdade de Imperatriz, Brasil

E-mail: samuelsilvalimaa@gmail.com

Renata da Conceição Oliveira

ORCID: https://orcid.org/0000-0002-2327-7606

Faculdade Pitágoras de Imperatriz, Brasil

E-mail: renata.jp2carrossel@hotmail.com

Cristiane de Oliveira Pinto

ORCID: https://orcid.org/0000-0003-4039-7455

Faculdade Pitágoras de Imperatriz, Brasil

E-mail: cristianelis25@gmail.com

Jonata Leal dos Santos

ORCID: https://orcid.org/0000-0001-7448-9900

Faculdade Pitágoras de Imperatriz, Brasil

E-mail: jonataleal.2@gmail.com

Mylleny Estrela Bandeira

ORCID: https://orcid.org/0000-0003-1734-1699

Faculdade Pitágoras de Imperatriz, Brasil

E-mail: mylleniestrelabandeira@ hotmail.com

Haylla Freitas Vieira

ORCID: https://orcid.org/0000-0002-3214-7037

Faculdade Pitágoras de Imperatriz, Brasil

E-mail: haylla29freitas@gmail.com

Wendel Chaves Carvalho

ORCID: https://orcid.org/0000-0001-8345-366X Faculdade Pitágoras de São Luís, Brasil

E-mail: wendelchavescarvalho@ hotmail.com

David Wilkerson dos Santos Silva

ORCID: https://orcid.org/0000-0001-5091-6752

Faculdade Pitágoras de Imperatriz, Brasil

E-mail: david_wilkerson15@hotmail.com

Lara Victória Dittz de Abreu Costa

ORCID: https://orcid.org/0000-0001-6193-0410

Universidade Federal do Espírito Santo, Brasil E-mail: lara-dittz@hotmail.com

Lorraine Ribeiro Santana

ORCID: https://orcid.org/0000-0002-7616-4517

Universidade Federal do Espírito Santo, Brasil

E-mail: lorraineribeiros@hotmail.com

Alfredo Carlos Rodrigues Feitosa

ORCID: https://orcid.org/0000-0003-4090-4760

Universidade Federal do Espírito Santo, Brasil E-mail: alfredofeitosaufes@gmail.com 


\begin{abstract}
Resumo
Objetivos: O objetivo desse estudo foi refletir e revisar sobre acerca da promoção de saúde bucal no contexto do ambiente escolar primário, descrevendo sobre os métodos de promoção de saúde no ensino primário e acerca dos programas de incentivo à promoção de saúde no ensino primário. Revisão de literatura: A condução da avaliação de saúde bucal realizada no ambiente escolar exige de modo inicial o reconhecimento de capacidades referentes a própria equipe de saúde, bem como dos profissionais da educação e que representem boa parte da estrutura da escola. Atualmente, o empenho da classe odontológica encontra-se voltado, principalmente, para a prevenção das doenças orais, o que representa um mecanismo muito mais simplório, com menos custos e sábio de atenção à saúde. Nessa perspectiva, destaca-se a necessidade do ensino e da motivação de hábitos de higiene bucal às crianças, considerando que os métodos de educação e motivação possuem a finalidade de esclarecer os indivíduos sobre as doenças orais assim como mudar seus hábitos de higiene. Considerações finais: A escola possui o papel de um ambiente social e educacional favorável no que se tange a se trabalhar conhecimentos e mudanças de comportamento. Nessa perspectiva, a compreensão das necessidades de crianças no ensino primário é de fundamental importância para que os profissionais de saúde e educação se empenhem, no intuito de influenciar de forma positiva essa população e atuar em conjunto a eles como multiplicadores de saúde.
\end{abstract}

Palavras-chave: Escolaridade; Odontologia; Política de saúde; Promoção da saúde.

\begin{abstract}
Objectives: The aim of this study was to reflect and review about oral health promotion in the context of the primary school environment, describing health promotion methods in primary education and health promotion incentive programs in primary education. Literature review: Conducting the oral health assessment carried out in the school environment requires, initially, the recognition of abilities related to the health team itself, as well as to education professionals, who represent a good part of the structure of the school. Currently, the commitment of the dental profession is mainly focused on the prevention of oral diseases, which represents a much simpler mechanism, with less cost and wiser healthcare. From this perspective, the need for teaching and motivating children in oral hygiene habits is highlighted, considering that the methods of education and motivation are intended to clarify individuals about oral diseases as well as to change their hygiene habits. Final considerations: The school has the role of a favorable social and educational environment when it comes to working knowledge and changes in behavior. From this perspective, understanding the needs of children in primary education is of fundamental importance for health and education professionals to commit themselves, in order to positively influence this population and act together with them as health multipliers.
\end{abstract}

Keywords: Dentistry; Health policy; Health promotion; Schooling.

\title{
Resumen
}

Objetivos: El objetivo de este estudio fue reflexionar y revisar sobre la promoción de la salud bucal en el contex to del entorno de la escuela primaria, describiendo los métodos de promoción de la salud en la educación primaria y los programas de incentivos de promoción de la salud en la educación primaria. Revisión de la literatura: La realización de la evaluación de salud bucal realizada en el ámbito escolar requiere, inicialmente, el reconocimiento de habilidades relacionadas con el propio equipo de salud, así como con los profesionales de la educación, quienes representan buena parte de la estructura de la escuela. Actualmente, el compromiso de la profesión odontológica se centra principalmente en la prevención de las enfermedades bucodentales, lo que representa un mecanismo mucho más sencillo, con menor coste y más sabio en la atención sanitaria. Desde esta perspectiva, se destaca la necesidad de enseñar y motivar a los niños en los hábitos de higiene bucal, considerando que los métodos de educación y motivación están destinados a esclarecer a las personas sobre las enfermedades bucodentales así como a cambiar sus hábitos de higiene. Consideraciones finales: La escuela tiene el papel de un entorno social y educativo favorable cuando se trata de conocimientos prácticos y cambios de comportamiento. Desde esta perspectiva, comprender las necesidades de los niños en la educación primaria es de fundamental importancia para que los profesionales de la salud y la educación se comprometan, con el fin de influir positivamente en esta población y actuar junto a ellos como multiplicadores de la salud.

Palabras clave: Enseñanza; Odontología; Política de salud; Promoción de la salud.

\section{Introdução}

A educação é considerada como a base principal presente no estímulo e na proteção da saúde (Garbin et al., 2012). A saúde bucal é parte essencial da saúde geral, podendo ser concebida como uma soma de situações objetivas (biológicas) e subjetivas (psicológicas), que proporcionam ao ser humano efetuar funções fundamentais inerentes ao sistema estomatognático, como: fonação, mastigação e deglutição, além disso, as pessoas visam a grandeza estética específica à região anatômica, no sentido de aperfeiçoar a autoestima e conviver socialmente sem inibição ou constrangimento (Mestriner et al., 
2017).

A escola é considerada um instrumento ideal para o desenvolvimento de estratégias que promovam saúde, devido a sua abrangência e ao fato de ser também responsável pela formação de atitudes e valores. Segundo a Organização Panamericana de Saúde (OPS), a promoção de saúde nas escolas trabalha com uma visão multidisciplinar do ser humano, responsável por considerar as pessoas em seu contexto familiar, comunitário, social e ambiental. Sendo assim, a educação em saúde bucal no âmbito escolar se constitui em um instrumento essencial para o desenvolvimento integral do estudante (Castro et al., 2012).

A promoção de saúde é considerada uma forma de se trabalhar a prevenção da cárie dental e de outros agravos atrelados a saúde bucal, sendo realizada preferencialmente em ambientes de convívio social, como as escolas, já que dessa forma, a incorporação de hábitos e comportamentos saudáveis, atrelados à saúde bucal, torna-se mais efetiva (Antunes, Antunes \& Corvino, 2008). Assim, a fase pré-escolar torna-se primordial para alcançar longevidade e eficiência nas ações que promovam saúde bucal, destacando o meio na qual os indivíduos estão vivendo, considerando que a existência de uma ligação proveniente da infância com a educação tem beneficiado a incorporação de medidas de promoção e prevenção em saúde bucal por meio de métodos acessíveis e didáticos para esse público (Garbin et al., 2012).

Dessa maneira, a presença do ensino nessa esfera, deve ser estabelecida assim que possível, pois, é nessa fase que é formada a base para a construção do conhecimento, sendo demonstrado através do comportamento, dos princípios e de hábitos. Consequentemente, a aprendizagem desses conhecimentos permitirá o andamento de habilidades e capacidades pessoais, possibilitando o desenvolvimento de comportamentos e a concepção de valores que acompanhem o indivíduo e o seu lar a atuarem, no seu cotidiano, em proveito da própria saúde oral (Shaghaghian; Bahmani \&Amin, 2015).

Nessa perspectiva, é importante ressaltar que esse modo não deve se enquadrar apenas na transmissão de conhecimentos, mas, no incentivo das práticas, como muito já foi alcançado dentro de uma metodologia interativa, no reconhecimento de mostrar uma saúde bucal saudável com maior clareza (Mestriner et al., 2017). Assim, o objetivo desse trabalho foi revisar acerca da promoção de saúde bucal no contexto do ambiente escolar primário, descrevendo sobre os métodos de promoção de saúde no ensino primário e acerca dos programas de incentivo à promoção de saúde no ensino primário.

\section{Metodologia}

Esse artigo é resultado de uma revisão da literatura narrativa sobre promoção da saúde no âmbito educacional infantil, com uma abordagem de pesquisa qualitativa que envolve: levantamentos de informações, estudos, leituras e análises de opiniões semelhantes e diferentes apontadas por diferentes autores, bem como a interpretação desses estudos de maneira a se perceber a relevância do assunto em questão, considerando que os artigos de revisão narrativa constituem publicações consideradas amplas, apropriadas na descrição e discussão do desenvolvimento ou do "estado da arte" de um determinado assunto, sob ponto de vista teórico e até mesmo contextual (ROTHER, 2007) e de acordo Pereira et al. (2018) que demonstrou que os métodos de pesquisa qualitativos são considerados aqueles nos quais se torna fundamental a interpretação por parte do pesquisador com suas opiniões sobre o fenômeno que se encontra em estudo.

Em virtude ao objetivo da pesquisa, análise da importância do âmbito educacional em propor atividades para conscientizar e promover a saúde bucal, o estudo foi desenvolvido em uma perspectiva qualitativa, através de uma revisão bibliográfica para o processo de construção do artigo. Foi realizada uma busca nas bases de dados Google Scholar e PubMed por meio do uso das palavras-chave "Assistência Integral à Saúde", "Criança", "Escolaridade", "Promoção da Saúde" e "Saúde Bucal", com seleção de fontes como artigos científicos publicados nos idiomas português e inglês, sem data limite de 
publicação. Assim, foram incluídos capítulos de livros, estudos clínicos, relatos de casos, trabalhos de conclusão de curso, dissertações e teses. Foram excluídos estudos realizados com animais, estudos in vitro, estudos laboratoriais e outros que estivessem fora da temática abordada. Após a identificação dos textos, realizou-se uma leitura e análise criteriosas a fim de se relacionar os textos que compuseram a revisão da literatura ao tema proposto.

\section{Resultados}

\section{Métodos de promoção de saúde no ensino primário e Programas de incentivo à promoção de saúde no ensino primário}

A introdução do conhecimento acerca de estratégias de educação e promoção de saúde devem ter início nas escolas de graduação dos profissionais de saúde. Assim é papel das escolas médicas estimularem os seus acadêmicos a participarem deste momento atual mundial, onde a sanidade humana é o objetivo principal, tanto em seus aspectos físicos, psicológicos, sociais, ambientais, históricos, culturais e espirituais. Sendo assim, nota-se que essa é a visão dita holística ou global do ser humano, sendo que todas elas têm a mesma importância para a sua sobrevivência (Martins e Silva et al., 2020).

Nesse contexto, a realização de ações com ênfase em educação em saúde se fundamenta na prevenção de agravos, os quais podem repercutir no processo saúde-doença, melhorando a qualidade de vida das pessoas envolvidas. Dessa forma, a aplicação desse tipo de medida de intervenção parece adequada a realidade do local de execução do projeto, considerando que a intenção fundamental desse era passar informações que potencialmente possam ser difundidas para toda comunidade. Assim, nesse processo de Educação em Saúde, fica evidente a necessidade da participação de 3 segmentos de extrema importância para o sucesso de qualquer ação: atores, gestores e população (Falkenberg, Mendes, Moraes, \& Souza,2014; Sacramento et al., 2020; Parente et al., 2020).

Inúmeros profissionais do âmbito odontológico pontuam cada vez mais a necessidade interdisciplinar de trabalhar em conjuntura com diversos profissionais que pertençam a área de educação, como, por exemplo, na própria área pedagógica. Essa união de profissionais acaba contribuindo para o melhoramento das condições de atendimento, mas, principalmente atende a uma classe muito importante - a criança - a qual consegue gradativamente adquirir hábitos e informações em sua fase inicial de aprendizagem, o que, seguindo as estimativas, reflete posteriormente em uma população de certa maneira mais consciente e informada acerca da importância dos métodos preventivos, antes mesmo da necessidade de tratamento (Toassi \& Petry, 2002; Aquilante et al., 2003; Alves et al., 2004).

Dessa maneira, a condução da avaliação de saúde bucal realizada no ambiente escolar exige de modo inicial o reconhecimento de capacidades referentes a própria equipe de saúde, bem como dos profissionais da educação e que representem boa parte da estrutura da escola. Isso acaba incluindo vários aspectos atrelados à preparação e disponibilidade da equipe para a realização da avaliação, do registro, avaliação e discussão integrada de resultados, assim como a mobilização e estrutura da escola para condução dessa ação (Ministério da Saúde, 2013).

Em suma, as ações de promoção da saúde são representadas pela educação em saúde, higiene bucal supervisionada e a aplicação tópica de flúor, sendo que apenas a última é de competência exclusiva do profissional de saúde bucal. Nessa perspectiva, a capacitação de professores e jovens de referência/cuidadores de saúde bucal na escola, a realização de atividades criativas como a produção de peças de teatro e pequenas apresentações sobre a temática, assim como o estímulo a visitas de aspecto regular ao cirurgião-dentista de acordo com a necessidade identificada para cada estudante podem ser ideias que podem compor o projeto de cuidado da saúde bucal na escola. Essas são alternativas criativas para proporcionar a continuidade das ações em promoção da saúde bucal (Ministério da Saúde, 2013). As crianças em idade pré-escolar apresentam maior capacidade para desenvolver hábitos saudáveis de higiene, quando motivadas, tornando esse comportamento resistente a mudanças. Nesse contexto, a manutenção das superfícies dentárias livres de biofilme deve ser almejada e formas para 
avaliação de tais condições devem ser trabalhadas, não só para o controle das ações, mas como método de estímulo para o desenvolvimento de autonomia em saúde (Aquilante et al., 2003).

A escovação dentária se constitui como a forma mais utilizada e socialmente aceita acerca de higiene bucal. No entanto, para ser eficaz combatendo à placa bacteriana, a escovação necessita ser direcionada e supervisionada pelo profissional, sendo fundamental a motivação do paciente na obtenção dos melhores resultados. Assim, a eliminação da placa bacteriana exige a aplicação de métodos mecânicos de higiene bucal, que possuem a necessidade de serem ensinados e treinados com as crianças. A maioria das pessoas sabe que para ter saúde bucal é necessário escovar os dentes diariamente. Mesmo assim, na maioria das vezes, a higiene bucal se torna deficiente. Em suma, as técnicas de controle da placa bacteriana necessitam de tempo e destreza e, consequentemente, o paciente só participa de maneira adequada quando bem motivado. Para que o paciente seja educado, torna-se importante que haja uma comunicação de forma efetiva entre o cirurgião-dentista e o próprio paciente considerando que sem esta comunicação, não há aprendizado (Sá Lo \& Vasconcelos, 2009).

Para esse tipo de avaliação, são preconizados indicadores, como o Índice de Higiene Oral Simplificado (IHOS). Por meio deste, aferem-se a motivação, o empenho e o cuidado do paciente durante o tratamento, utilizando a quantidade de biofilme presente nos elementos dentais (Ministério da Saúde, 2013). Assim, nota-se que é de extrema importância que todos os momentos que envolvem a avaliação das condições de saúde representem referências acerca de possíveis pontos de discussão que possam reverter em conhecimentos pedagógicos, assim como estarem previstos no dia-a-dia da escola e, consequentemente, incluídos na estrutura do projeto político pedagógico. Inúmeras estratégias como a disponibilização de alimentação saudável na escola através de parcerias de cunhos institucionais e comunitárias e construção de hortas escolares; a adequação do ambiente físico da escola com a disponibilização de espaços ideais para alimentação e a realização de práticas de higiene corporal e bucal·; bem como o desenvolvimento de estratégias lúdicas e recreativas; além de práticas de educação para saúde voltadas para o desenvolvimento da autonomia, representam exemplos de estratégias que podem favorecer a promoção da saúde geral e bucal na escola e que se fortalecem quando fazem parte do Projeto Político Pedagógico da escola (Kusma; Moysés \& Moysés, 2012).

Por conseguinte, como uma maneira de garantir a sustentabilidade dessas ações é necessário, também, que a própria equipe de educação e saúde esclareça os objetivos acerca das ações de saúde bucal do PSE, para todos que estejam envolvidos na ação (profissionais de saúde, da escola, merendeiras, educandos, pais e responsáveis) e buscar integrar esses profissionais no planejamento, execução, avaliação e cuidado que serão realizados (Ministério da Saúde, 2013).

Essa integração é uma estratégia de fundamental importância que pode oportunizar o reconhecimento de problemas, seus determinantes e fatores de risco associados, podendo favorecer o empoderamento tanto individual quanto coletivo. Dessa forma, esse envolvimento também acaba reforçando a importância da participação de todos no cuidado com a saúde bucal. Além disso, o momento da avaliação de saúde bucal pode constituir um importante momento de mobilização coletiva em prol de práticas educativas que possam favorecer o aprendizado acerca das estratégias necessárias nesse ambiente comunitário (Carvalho et al., 2013).

Assim, a frequência da realização das ações direcionadas à aplicação tópica de flúor e escovação supervisionada acaba sendo determinada pelos resultados que são obtidos na avaliação da saúde bucal de todos os estudantes. Nessa perspectiva, a ação de escovação supervisionada realizada por profissionais da saúde (escovação supervisionada direta) idealmente deve ocorrer pelo menos duas vezes ao ano e a realizada por profissionais educadores (escovação supervisionada indireta), podendo ocorrer mais vezes no cotidiano escolar (Kusma; Moysés \& Moysés, 2012).

Nessa perspectiva, dentre os instrumentos disponíveis, destacam-se as atividades de acrósticos, textos enigmáticos, textos informativos, músicas, técnicas de higiene oral, desenhos, redação e avaliação do projeto pelas próprias crianças, atividades de ligar palavras a figuras, estórias em quadrinhos, jogos de memória, cruzadinhas, poemas, dramatizações, 
fantoches e escrita espontânea. Conforme as atividades que utilizaram o acróstico, algumas instruem as crianças a formarem um texto a partir dele, dando sentido à atividade, podendo o avaliador constatar se as crianças adquiriram os conceitos. O texto enigmático proporciona à criança escrever um texto e refletir sobre um determinado assunto, podendo, assim, se conscientizar. A adaptação de também é uma atividade muito valorizada, pois explora o conceito e a prática da musicalidade, os versos, palavras diminutivas e pode-se, por meio delas, obter-se conhecimentos sobre conceitos em saúde bucal. Infere-se que escolha de atividades adequadas para determinadas faixas etárias é relevante. De igual importância é a orientação que o professor dá ao solicitar uma atividade (Júnior \& Mialhe, 2008).

Nota-se que têm se tornado imprescindíveis a criação e o incentivo de programas educativos que ampliem a promoção de saúde, já que atividades como estas são capazes de motivar as crianças, em fase pré-escolar, para mudança de hábitos, estimulando as mesmas para o cuidado com a sua saúde bucal. É importante enfatizar que esse processo educativo ocorre de forma lenta; por isso, deve ser contínuo para que alterações precoces de maus hábitos e comportamentos sejam capazes de transformar essa realidade (Migliato et al., 2008).

No Brasil, a Pesquisa Nacional de Saúde do Escolar (PeNSE) foi constituída com base no primeiro inquérito nacional referente a escolares, atendendo aos objetivos de implantação do Sistema Nacional de Monitoramento da Saúde do Escolar. A PeNSE tem como objetivo subsidiar gestores e profissionais de saúde com dados informativos que sejam confiáveis para a orientação e avaliação de um conjunto de políticas de saúde destinadas aos adolescentes. Assim na PeNSE foram coletadas informações sociodemográficas, a respeito de alimentação, imagem corporal, atividade física, tabagismo, consumo de álcool e outras drogas, saúde bucal, comportamento sexual, violência, acidentes, segurança e medidas antropométricas, tendo sido o módulo de saúde bucal foi composto por duas questões, referentes à frequência de escovação dentária e relato de dor dentária (Pense, 2009).

Reconhecendo a escola como um espaço com privilégios para a disseminação de promoção de saúde, em 2007 foi instituído no Brasil o Programa Saúde na Escola (PSE) através de decreto ministerial (decr. $\mathrm{n}^{\circ}$ 6.286), em cujo artigo $4^{\circ}$ consta: "As ações em saúde previstas no âmbito do PSE considerarão a atenção, promoção, prevenção e assistência, e serão desenvolvidas articuladamente com a rede de educação pública básica e em conformidade com os princípios e diretrizes do SUS, podendo compreender as seguintes ações, entre outras:" item V - "avaliação da saúde e higiene bucal". Dessa forma, esse programa busca contribuir para o fortalecimento de diversas ações que possam integrar as áreas de Saúde e Educação no enfrentamento de vulnerabilidades e que ampliem a articulação a respeito de saberes e experiências presentes no planejamento, na realização e na avaliação das iniciativas para alcançar o desenvolvimento integral dos estudantes da rede pública de educação básica e que deem suporte para o processo formativo dos profissionais de saúde e educação de forma permanente e continuada (Moretti et al., 2010).

\section{Discussão}

A promoção em saúde e o desenvolvimento de questões que possam circundar o tema da saúde são importantes no sentido de serem abordadas várias áreas em rede, inclusive com a integração da escola, por meio de suas disciplinas, na promoção de conteúdos adequados, metodologias e abordagem integrada e instrumentos operacionais multidisciplinares validados em sua eficácia para serem desenvolvidos com os estudantes, professores, pais e demais membros da comunidade escolar (Lima, Malacarne \& Strieder, 2012). O cenário escolar deve ser entendido como um laboratório de promoção da saúde, e as ciências da saúde devem preenchê-lo através da implementação de projetos que ponham em pauta o autocuidado e a promoção para o viver saudável (Carraro et al., 2019). Assim, a escola não deve atuar apenas no ensino das matérias 
disciplinares tradicionais e sim, agir como um multiplicador dos hábitos sociais, de higiene e de prevenção, tratando o aluno por sua integralidade e o envolvendo neste processo como um corresponsável (Morais et al., 2020).

Com relação à saúde oral, autores relatam que a higiene bucal só se consolidará como um hábito quando estiver na idade adulta reduzindo a ocorrência de doenças da cavidade oral. Desse modo, o instituto escolar tem sido considerado um espaço ideal para a expansão de métodos e práticas que promovam saúde, devido sua ex tensão e a autenticidade de ser também encarregado pela construção de conduta e princípios. Com esse intuito, os programas devem ser cautelosamente elaborados, tendo em vista, não só a idade de seus participantes, como também sua realidade social, econômica e cultural (Valarelli et al., 2011).

A saúde oral no ensino primário compõe parte da saúde pública e, para ser efetiva, precisa estar voltada idealmente para três fatores imprescindíveis: vontade política, infraestrutura social e educação em saúde. Assim, a maneira mais eficaz de desenvolver programas educativos preventivos de saúde nas escolas acontece através do trabalho em conjunto tratando-se profissionais de saúde e educação. Dessa forma, toda escola, até mesmo aquela que possua recursos escassos, uma vez administrada através de políticas de encorajamento à cidadania, consegue atuar na promoção de saúde e torna-se uma excelente ideia operacional, podendo atuar como força de união para melhorar a saúde e a educação (Taglietta et al., 2011).

Atualmente, o empenho da classe odontológica encontra-se voltado, principalmente, para a prevenção das doenças orais, o que representa um mecanismo muito mais simplório, com menos custos e sábio de atenção à saúde. Nessa perspectiva, destaca-se a necessidade do ensino e da motivação de hábitos de higiene bucal às crianças, considerando que os métodos de educação e motivação possuem a finalidade de esclarecer os indivíduos sobre as doenças orais assim como mudar seus hábitos de higiene (Garbin et al., 2009). Nota-se que em odontologia, os procedimentos educativos apresentam uma finalidade básica, modificar o comportamento de saúde bucal dos indivíduos, pois, uma vez educados, os pacientes tornam-se receptivos e cooperadores com as medidas que lhes são prescritas (Santos, Rodrigues \& Garcia, 2002).

Nesse sentido, nossos achados corroboram com Cortellazzi (2006) que constatou em seu estudo que o nível de escolaridade materna se encontra relacionado a aspectos de cunho comportamental, ou seja, relacionados a um grau elevado de instrução que se torna de fundamental relevância na transmissão de conhecimentos aos filhos a respeito de educação em saúde bucal, tais como higienização dentária, orientação da dieta e até mesmo consumo de açúcar. Desse modo, pode-se notar que crianças que persistem em suas casas durante toda a infância, recebendo cuidados de pais e responsáveis com baixos níveis escolaridade, além de escassa informação a respeito de higiene oral e com uma alimentação com altos níveis de açúcar possuem geralmente um índice de cárie dental maior que as que recebem atenção especial em seus ambientes escolares de maneira adequada.

Por fim, este estudo realça a importância dos programas de educação e de promoção de saúde oral nas escolas, além de considerar a fundamental importância do acompanhamento diário pelos agentes escolares de saúde dadas as limitações existentes nessa área até os dias de hoje. Nessa perspectiva, esse fato é concordante com alguns estudos que demonstram que programas educativos acerca de higiene oral devem ser incluídos na educação escolar das crianças, considerando que a faixa etária primária de quatro a sete anos é considerada a época mais oportuna para que as crianças desenvolvam inúmeros hábitos alimentares e de higiene adequados, levando em consideração que os modelos de comportamento aprendidos nessa idade são profundamente fixados e resistentes a alterações ao longo do tempo (Taglietta et al., 2011).

\section{Conclusão}

A promoção de saúde é considerada uma estratégia fundamental presente no processo de formação de comportamentos que promovam e mantenham a saúde, devendo ser efetiva no aperfeiçoamento do conhecimento dos 
indivíduos. Assim, a escola possui o papel de um ambiente social e educacional favorável no que se tange a se trabalhar conhecimentos e mudanças de comportamento. Nessa perspectiva, a compreensão das necessidades de crianças no ensino primário é de fundamental importância para que os profissionais de saúde e educação se empenhem, no intuito de influenciar de forma positiva essa população e atuar em conjunto a eles como multiplicadores de saúde.

Inúmeras evidências demonstram que a motivação e a educação em saúde são ferramentas extremamente importantes quando são empregadas de maneira leve, atrativa e eficaz para transmissão de informações, constituindo dessa maneira o objetivo em si da promoção de saúde nas escolas. Em suma, a literatura enfatiza que a escola é um ambiente favorável para a aplicação de diversos programas de educação em saúde por estar inserida em todas as dimensões a respeito do aprendizado. Dentro desse contexto, os resultados dessa presente revisão narrativa proporcionam evidências científicas dos métodos de promoção de saúde bucal em ambiente escolar primário tratando-se de educação em saúde nas escolas, como estratégia para diminuição dos índices de doenças atreladas a esses fins como, por exemplo, a cárie dentária e doenças periodontais em escolares primários.

São necessários mais estudos que possam corroborar com esses achados, trazendo novas alternativas e visões a respeito da promoção em saúde bucal nas escolas, seja para essa faixa etária assim como outras que compõem o ambiente escolar. Dessa forma, poderemos refletir acerca da necessidade de ressaltar a importância da inserção desse conteúdo no ambiente escolar, levando em consideração que a educação em saúde e a própria adoção de hábitos como higienização bucal e alimentação saudável devem idealmente compor o cotidiano escolar desde a pré-escola. Assim, os resultados desta pesquisa foram obtidos mediante uma revisão bibliográfica que revelou pontos que ainda são necessários intervir em programas de educação em saúde bucal voltados para as crianças na fase da pré-escola.

\section{Referências}

Alves, M. A, Volschan, B. C. G, Haas, N. A. T. Educação em saúde bucal: sensibilização dos pais de crianças atendidas na clínica integrada de duas Universidades privadas. (2004). Pesq Bras Odontoped Clin Integr., 4 (1), 47-51.

Antunes L. S., Antunes, L. A. A., Corvino, M. P. F. (2008). Percepção de pré-escolares sobre saúde bucal. Rev Odontol Univ. Cid São Paulo, 20,52 - 59.

Aquilante, A. G., Almeida, B. S., Castro, R. F. M., Xavier, C. R. G., Peres, S. H. C. S \& Bastos, J. R. M. (2003). A importância da educação em saúde pública para pré-escolares. Rev Odontol UNESP., 32, 39 - 45.

Carraro, E. C., Militão, E, C.\& Viana, H. B. (2019). A percepção dos educadores quanto ao seu papel na promoção de hábitos e estilos de vida saudáveis no ambiente escolar. Rev. Fac. Educ. (Univ. do Estado de Mato Grosso), p.1-24.

Carvalho, T. H. L., Pinheiro, N. M. S., Santos, J. M. A. \& Costa, L. A. D. et al. (2013). Estratégias de promoção de saúde para crianças em idade pré-escolar do município de Patos - PB. Revista de Odontologia da UNESP, 42 (6), 426 - 431.

Castro, C. O., Oliveira, K. S., Carvalho, R. B., Garbin, C. A. S. et al. Programas de educação e prevenção em saúde bucal nas escolas: análise crítica de publicações nacionais. Odontologia Clínico-Científica, 11 (1), 51 - 56.

Cortellazzi, K. L. Indicadores de risco de cárie dentária e de gengivite em crianças na faixa etária de 5 anos. (2006). Faculdade de Odontologia de Piracicaba. Piracicaba.

Falkenberg, M. B., Mendes, T. de P. L., Moraes, E. P. \& Souza, E. M. (2014). Educação em saúde e educação na saúde: conceitos e implicações para a saúde coletiva. Ciência \& Saúde Coletiva,19(3), 847-852.

Garbin, C. A. S., Garbin A. J. I., Santos K. T. \& Lima D. P. (2009). Oral health education in schools: promoting health agents. Int J Dent Hygiene, 7, 212 216.

Instituto Brasileiro de Geografia e Estatística. Pesquisa Nacional de Saúde do Escolar (PeNSE). (2009). http://www.ibge.gov.br/home/estatistica/populacao/pense/pense.pdf

Júnior, M. M. \& Mialhe, F. L. (2008). A importância da professora na promoção da saúde bucal dos escolares. Revista de Odontologia da Universidade Cidade de São Paulo, 20 (1).

Kusma, S. Z., Moysés, S. T. \& Moysés S. J. (2012). Promoção da saúde: Perspectivas avaliativas para a saúde bucal na atenção primária em saúde. Cadernos de Saúde Pública, 28. 
Research, Society and Development, v. 10, n. 12, e78101220191, 2021 (CC BY 4.0) | ISSN 2525-3409 | DOI: http://dx.doi.org/10.33448/rsd-v10i12.20191

Lima, D. F., Malacarne, V. \& Strieder, D. M. O papel da escola na promoção de saúde - uma medicação necessária. (2012). Revista Científica Eccos, 28, 191 -206 .

Martins e Silva, E. S. M., Ono, B. H., Souza, J. C., Menin, I. B. F. et al. (2020). Mídia e promoção da saúde em tempos de COVID-19. Research Society and Development, v.9, n.8, 2020.

Mestriner, S. F., Mesquita, L. P., Júnior, W. M. \& Bulgarelli, A. F. (2017). Percepções de estudantes de Odontologia sobre a experiência em um estágio não obrigatório no SUS. Revista da ABENO, v.17, n.4, 171 - 182.

Migliato K. L., Mendes E. R., Souza L.Z., Cortellazzi L. L, Mistro F.Z., Paganini G.A., et al. (2018). Avaliação de um programa preventivo-educativo desenvolvido entre a UNIARARAS e a Usina São João de Araras, SP. RFO, 13, 14 - 18.

Ministério da Saúde. Ministério da Educação. (2013). Promoção da Saúde Bucal. Cadernos temáticos do PSE - 7. Editora MS.

Morais, S. R., Freitas, V. J. G., Alves, L. S. B., Nóbrega, C. B. C. et al. (2020). O papel da extensão universitária na capacitação de professores como agentes multiplicadores da saúde bucal. Research, Society and Development, 9 (8).

Moretti, A. C., Teixeira, F. F., Suss, F. M. B. \& Lawder, J. A. C. et al. (2010). Intersetorialidadade nas ações de promoção de saúde realizadas pelas equipes de saúde bucal de Curitiba (PR). Ciência \& Saúde Coletiva, 15 (1).

Parente, F. S., Oliveira, R. C. M.., Santos, L. C. M., Tsukimata, M. Y., Silva, J. M. R., Sacramento, R. C., Vasconcelos, L. A., Pereira, C. E. A., Sousa, J. S., Lopes, L. J. S. (2020). Health Education: a socio-educational tool for health promotion for children in a public school in Belém do Pará, Brazil. Research, Society and Development, 9 (7): 1-13

Pereira, A. S., Shitsuka, D. M., Parreira, F. J., Shitsuka R. Metodologia da pesquisa científica. (2018). Universidade Federal de Santa Maria. Santa Maria.

Rother, E. T. (2007). Revisão sistemática x revisão narrativa. Acta Paulista de Enfermagem, 20 (2).

Sá, L. O. \& Vasconcelos M. M. V. B. (2009). A Importância da educação em saúde bucal nas escolas de Ensino Fundamental - Revisão de literatura. Odontologia Clín-Científic, 8(4), 299-303.

Sacramento, R. C., Vasconcelos, L. A., Neves, A. D. R., Souza, B. S. L. Okada, E. S. T., Costa, F. R. N., ...Santos, B. R. F. (2020). Ações de Enfermagem na Promoção da Saúde da Mulher no Contexto Amazônico. Research, Society and Development, 9(5), 171953319.

Santos, P. A., Rodrigues, J. A. \& Garcia, P. P. N.S. (2002). Avaliação do conhecimento dos professores do ensino fundamental de escolas particulares sobre saúde bucal. Rev. Odontol. UNESP, .31(2), $205-214$.

Shaghaghian, S., Bahmani, M. \& Amin M. (2015). Impact of oral hygiene on oral health-related quality of life of preschool children. Int J Dent. Hyg.

Taglietta, M. F. A., Bittar, T. O., Brandão, G. A. M. \& Vazquez, F. L. et al. (2011). Impacto de um programa de promoção de saúde escolar sobre a redução de prevalência da cárie em crianças pré-escolares de Piracicaba-SP. RFO UFP, 16 (1), 13 - 17.

Toassi, R. F. C \& Petry, P. C. (2002). Motivação no controle do biofilme dental e sangramento gengival em escolares. Rev Saúde Pública, 36 (5), $634-637$.

Valarelli, F. P., Franco, R. M., Sampaio, C. C. \& Mauad, C. Importância dos programas de educação e motivação para saúde bucal em escolas: relato de experiência. Odontologia Clínico-Científica, 10 (2), 173 - 176. 